\title{
Memory Contextualization: The Role of Prefrontal Cortex in Functional Integration across Item and Context Representational Regions
}

\author{
Wei Zhang ${ }^{1,2}$, Vanessa A. van Ast $^{3}$, Floris Klumpers ${ }^{1,2}$, Karin Roelofs ${ }^{1,2 *}$, \\ and Erno J. Hermans ${ }^{1,4 *}$
}

\begin{abstract}
Memory recall is facilitated when retrieval occurs in the original encoding context. This context dependency effect likely results from the automatic binding of central elements of an experience with contextual features (i.e., memory "contextualization") during encoding. However, despite a vast body of research investigating the neural correlates of explicit associative memory, the neural interactions during encoding that predict implicit context-dependent memory remain unknown. Twentysix participants underwent fMRI during encoding of salient stimuli (faces), which were overlaid onto unique background images (contexts). To index subsequent context-dependent memory, face recognition was tested either in intact or rearranged con-
\end{abstract}

\section{INTRODUCTION}

Context is essential for memory retrieval. It is well established that memories are easier to recall when retrieval occurs in a context that resembles the original encoding context (van Ast, Cornelisse, Meeter, \& Kindt, 2014; van Ast, Cornelisse, Meeter, Joëls, \& Kindt, 2013; Smith \& Vela, 2001; Godden \& Baddeley, 1975; Tulving \& Thomson, 1973). Memory contextualization, in which an event and its context are automatically bound together into one integrated representation during encoding, is vital for the subsequent retrieval of relevant memories in specific situations (van Ast et al., 2013, 2014; Liberzon \& Sripada, 2008). Conversely, the inability to properly contextualize information has been linked to fragmentation of episodic memories and overgeneralization of (fear) memories that are characteristic of, for instance, posttraumatic stress disorder (PTSD; Quaedflieg et al., 2015; Acheson, Gresack, \& Risbrough, 2012; Meyer et al., 2012; Brewin, Gregory, Lipton, \& Burgess, 2010; Liberzon \& Sripada, 2008; Ehlers \& Clark, 2000) or schizophrenia (Talamini, de Haan, Nieman,

\footnotetext{
${ }^{1}$ Donders Institute for Brain, Cognition, and Behaviour, ${ }^{2}$ Behavioural Science Institute, Radbound University, ${ }^{3}$ University of Amsterdam, ${ }^{4}$ Radboud university medical center *These authors contributed equally to this article.
}

texts, after scanning. Enhanced face recognition in intact relative to rearranged contexts evidenced successful memory contextualization. Overall subsequent memory effects (brain activity predicting whether items were later remembered vs. forgotten) were found in the left inferior frontal gyrus (IFG) and right amygdala. Effective connectivity analyses showed that stronger context-dependent memory was associated with stronger coupling of the left IFG with face- and place-responsive areas, both within and between participants. Our findings indicate an important role for the IFG in integrating information across widespread regions involved in the representation of salient items and contextual features.

Linszen, \& Meeter, 2010). However, despite its relevance for general healthy memory function and clinical memory overgeneralization, the brain mechanisms underlying memory contextualization - the process whereby automatic encoding of an item-in-context results in subsequent implicit context effects on memory (i.e., memory enhancement by context similarity without testing for the explicit itemcontext association) — are just beginning to be explored.

Evidence from rodents shows that selective hippocampal damage results in deficits in forming a memory of the context (or location) where items were previously encountered (Eichenbaum, 2004). For instance, rats with hippocampal lesions fail to recognize a previously encountered object when contextual information relative to encoding has been changed (Mumby, Gaskin, Glenn, Schramek, \& Lehmann, 2002). Other evidence for a role of the hippocampus in implicit context effects on memory for cues comes from fear conditioning studies: In addition to conditioned freezing to an auditory cue, rats also exhibit freezing behavior when placed in the training context, but hippocampal lesions eliminate such contextual fear responses without affecting conditioned responses to the tone (Phillips \& LeDoux, 1992). Recently, using large-scale neuronal population recordings, new insights into the nature of hippocampal context representations have emerged. Such studies show that hippocampal 
ensemble context codes become associated with the memories and behaviors that are appropriate for that context. When confronted with a familiar context, the relevant hippocampal context code is automatically reexpressed, thereby priming the appropriate memories while reducing interference from memories associated with other contexts (for a review, see Smith \& Bulkin, 2014).

Analogous to this animal work, implicit context dependency of memories has been demonstrated in human studies where context similarity between the original encoding and retrieval context enhanced both recognition and recollection (Cox, Tijdens, Meeter, Sweegers, \& Talamini, 2014; van Ast et al., 2013, 2014; Talamini \& Gorree, 2012; Talamini et al., 2010; Tsivilis, Otten, \& Rugg, 2001). The consistent observation of highly context-dependent memories across this wide range of studies underscores contextdependent memory as one of the hallmarks of human episodic memory (Tulving, 1972). With respect to the neural correlates of such context-dependent memories, one study using magnetoencephalography has shown that, with an intact encoding-retrieval context, high theta power during encoding predicted successful recognition, whereas high theta power was detrimental when the retrieval context was rearranged relative to encoding. In addition, crossfrequency coupling analysis revealed a context-dependent theta-to-gamma memory effect, which was assigned to the left hippocampus using source localization (Staudigl \& Hanslmayr, 2013). An intracranial electroencephalography (iEEG) study furthermore implicated the prefrontal cortex (PFC) in successful item in context (temporal, in this case) binding during memory encoding, resulting in implicit context-dependent (i.e., clustering) memory (Long \& Kahana, 2015). However, given inherent uncertainty of (deep) source localization of EEG and magnetoencephalography signals and limited coverage of intracranial electroencephalography, an extension of these findings using techniques that allow for stronger spatial inferences, such as fMRI, is needed.

In contrast with such studies assessing (neural) encoding mechanisms that can subsequently modulate implicit context effects on recognition, previous fMRI studies have almost exclusively focused on how subsequent explicit memory of relations among cues is accomplished during encoding (Davachi, 2006). "Context" in these studies refers to scenes that were explicitly associated with objects: The corresponding retrieval tests directly probed associative memory among items and contexts. These studies converge on the idea that encoding of items versus context memories rely on distinct operations within the medialtemporal lobe, in which "what" and "where" processing streams function in parallel and converge within the hippocampus (Eichenbaum, Sauvage, Fortin, Komorowski, \& Lipton, 2012; Ranganath, 2010b; Diana, Yonelinas, \& Ranganath, 2007; Davachi, 2006). In addition to the hippocampus, PFC has been shown to play an equally important role in relational memory encoding when the integration of contextual information with specific item features is re- quired (Murray \& Ranganath, 2007; Summerfield et al., 2006; Prince, 2005). Indeed, lateral areas of PFC might be involved in selecting task-relevant information and in strategy implementation to find associations among items during memory encoding (Ranganath, 2010a). When an event is encoded in a particular context, PFC is therefore likely to contribute to the process of integrating relevant elements together. In agreement with this line of reasoning, some fMRI studies documented the involvement of both the hippocampus and PFC in enhanced item (i.e., word) memory in semantic versus nonsemantic contexts (Wagner, 1998; Kapur et al., 1994) or maintaining a representation of temporal context (Davachi \& DuBrow, 2015; Jenkins \& Ranganath, 2010). However, as these studies employed either encoding instructions informing about a subsequent explicit associative recognition test or used very broad context manipulations, such observations do not necessarily imply that the same brain regions are involved when no instructions are given to memorize the relevant items (i.e., incidental encoding) or when subsequent recognition is putatively modulated by implicit context effects (i.e., when memory enhancement by context similarity is assessed on item recognition without testing for the explicit item-context association). Indeed, a patient study suggests that the neural structures underlying explicit binding versus implicit contextual probing may differ (Graf \& Schacter, 1985; but see Chun \& Phelps, 1999). Thus, previous fMRI studies did not unequivocally address the question on which neural processes during encoding contribute to subsequent implicit context effects on memory.

Only two studies so far used an implicit context manipulation during recognition (Hayes, Baena, Truong, \& Cabeza, 2010; Hayes, Nadel, \& Ryan, 2007). In those studies, faces during encoding were presented against a naturalistic scene (Hayes et al., 2007, 2010) or a monocolored white (Hayes et al., 2007) and black (Hayes et al., 2010) background. Both studies found a decrement in recognition when a face encoded in a scene-context was presented against a monocolored background during recognition, as compared with faces that were presented against a monocolored background both during encoding and recognition. This effect was associated with a larger subsequent memory effect (SME), for example, in the hippocampus as well as enhanced connectivity between the hippocampus and visual association areas for the face in scene-context (Hayes et al., 2010). However, the stronger involvement of the hippocampus and its associated connectivity with face-responsive areas can be explained by the more complex and visually richer scene processing during encoding, as simply perceiving visually rich scenes has been associated with extensive hippocampal activation (Zeidman, Mullally, \& Maguire, 2015). Therefore, it remains to be convincingly shown that PFC and hippocampus are involved in memory contextualization during encoding, resulting in subsequent implicit context effects on memory. 
In the current study, we investigated the hypothesis that information across neural circuits involved in item and context representations would be integrated by the hippocampus and prefrontal regions to subserve memory contextualization, subsequently resulting in context-dependent memories. To investigate this, we implemented a memory task using neutral face images as items and scene images as contexts. During recognition, we asked our participants to indicate whether they had seen a given face image during encoding or not. Crucially, we manipulated context similarity during recognition relative to encoding by presenting faces against either identical (intact) or different (rearranged) scenes. By doing so, we ensured the presence of visually rich background images at all time. Furthermore, any modulation in memory performance by a shift in context cannot be driven by a change in familiarity, as all items and contexts have already been presented during encoding. Because stronger context-dependent memory is likely observed with "deeper" encoding strategies (De Beni \& Pazzaglia, 1995; Graf \& Schacter, 1985; Richardson, 1980), we instructed our participants to actively form a vivid mental image of the face in context and to indicate on a trial-by-trial basis how well they did in forming this mental face-in-context image. We expected that this "subjective memory contextualization index" during encoding would predict subsequent context-dependent face memory. Importantly, we did not inform our participants of the subsequent memory test, as to mimic real-life memory formation, and to prevent deliberate encoding of the explicit association between the face and the context. Behaviorally, we expected to observe enhanced face recognition in intact contexts versus rearranged contexts (Meyer, Krans, van Ast, \& Smeets, 2017; Cox et al., 2014; van Ast et al., 2013, 2014; Talamini et al., 2010). At the neural level, regardless of context, we expected to find SMEs for the faces in the faceresponsive region of the fusiform gyrus (FG; i.e., fusiform face area [FFA]) and/or the amygdala, in line with previous research (Kanwisher \& Yovel, 2006; Kanwisher, McDermott, \& Chun, 1997). In response to the presented contexts, we expected activity in the place-responsive region in the parahippocampal gyrus (PHG; i.e., parahippocampal place area [PPA]), as this region has been linked to the representation of contextual features (Epstein, Harris, Stanley, \& Kanwisher, 1999). Most importantly, we predicted that the integration of face (FFA/amygdala) and context (PPA) representations during effective memory contextualization would be associated with stronger neural activity in PFC and hippocampus. Finally, as actively integrating information likely requires functional connections among these brain regions, we also expected to find stronger neural coupling between PFC/hippocampus and distributed areas involved in representations of face (FFA/amygdala) and context (PPA) to support memory contextualization.

\section{METHODS}

\section{Participants}

Thirty-four right-handed university students (mean age $=$ 23.65 years) with no history of neurological or psychiatric disease gave written informed consent. Because of technical failure (e.g., MR scanner malfunction), data of eight participants were lost. Also, in line with previous memory research (Rimmele, Davachi, \& Phelps, 2012), participants were excluded from the analyses if their memory performance did not exceed chance level, leading to exclusion of an additional five participants (see Behavioral Data Analysis). Consequently, current analyses are based on 21 participants (mean age $=24.24$ years, $S D=2.86$; 15 women). All study procedures were approved by the local institutional review board (Commissie Mensgebonden Onderzoek Regio Arnhem-Nijmegen).

\section{Experimental Tasks and Procedure}

A localizer task and an encoding part of the memory contextualization task (MCT-encoding) were administered during fMRI scanning. Recognition (MCT-recognition) was assessed outside the scanner. In total, 280 color images of faces from three databases (Langner et al., 2010; Tottenham et al., 2009; Lundqvist, Flykt, \& Ohman, 1998) and 160 color images from various sources that depict indoor scenes, city landscapes, or natural scenes were selected for these two tasks. All tasks were administered using Presentation software (Version 16.4, www.neurobs. com).

\section{Localizer Task}

A functional localizer task was used to identify brain activation elicited specifically by faces and contexts. Forty face images (out of total 280), 40 context images (out of total 160), and an equal number of those images that were phase scrambled were presented in the task. Scrambling was accomplished within MATLAB (The MathWorks, Inc., Natick, MA) by randomizing the Fourier-transformed phase of R, G, and B layers of each stimulus image, which was then added to the existing RGB phase structures in the original images. Thereby, the relative phase of the RGB layers in the scrambled images was identical to that in the original images, and the color composition was kept the same as in the original images as well.

During the task, the four categories (face, context, scrambled face, and scrambled context) of image stimuli were presented with a blocked design. The order of the 16 blocks was mirrored to avoid covariation of task effects with linear trends. Within each block, 20 images from one specific category (e.g., face) were continuously presented in 20 trials of 1 -sec duration, without intertrial interval (ITI). In each trial, the face or scrambled face stimuli were presented within an oval shape in the middle of the screen, whereas the context or scrambled context 
stimuli were presented full screen. All images used in the localizer task were presented twice, resulting in a total of 320 trials. To keep participants engaged, a small red dot was presented in half of the trials that were randomly selected. Participants were instructed to indicate their detection of the red dot using a button press.

\section{Memory Contextualization Task}

During the memory contextualization task (MCT), faces served as to-be recognized items, whereas background scenes served as context. A total number of 240 face images and 120 context images were used in MCT. The face images were divided into two sets, each of which was randomly selected as either the target stimuli (i.e., used in both the encoding [MCT-encoding] and recognition [MCT-recognition] phases) or as the lures (i.e., used only in MCT-recognition phase). The 120 context images used in the MCT-encoding were used again in the MCTrecognition.

MCT-encoding. To pair the face and context images and to assign the paired face-context combinations to different (retrieval) conditions that were later presented in the MCT-recognition as either intact or rearranged, the randomly selected 120 face images for encoding were divided into two subsets with 60 gender-matched face images in each subset. Similarly, all context stimuli were also divided into two subsets that were matched on location (indoors vs. outdoors) of contexts. For each participant, the face and the context stimuli subsets were randomly assigned to the intact or rearranged retrieval condition of MCT-recognition and those faces and contexts were then randomly paired (i.e., into face-context combinations), resulting in randomized pairing of facecontext combinations within each condition. Furthermore, restrictions were made so that no trials from one condition (i.e., intact) were presented more than twice consecutively for each participant (see MCT-recognition). All stimuli were presented in 120 trials with a jittered ITI (average duration $=2 \mathrm{sec}$ ).

It is worth mentioning that the terms "intact" and "rearranged" have previously been used to refer to explicit knowledge of item-item associations in some studies (e.g., Litman \& Davachi, 2008; Giovanello, Schnyer, \& Verfaellie, 2004; Jackson \& Schacter, 2004). Here, we follow other recent studies that used the same terms to investigate implicit context effects (Meyer et al., 2017; van Ast et al., 2013, 2014; Hayes et al., 2010; Tsivilis, Otten, \& Rugg, 2001).

The MCT-encoding task was introduced as a test of imagination ability to induce deep incidental encoding (van Ast et al., 2013) since deeper or more vivid encoding strategies have been shown to strengthen contextdependent memory (De Beni \& Pazzaglia, 1995; Richardson, 1980). Specifically, participants were instructed to imagine a scene where the person (face) interacts with the place (context) as vividly as possible in each trial. We reasoned that these instructions would aid deep encoding and thereby promote the formation of an association between faces and their unique contexts (De Beni \& Pazzaglia, 1995; Richardson, 1980).

A 6-min long practice session, using the same face and context stimuli as in the localizer task was carried out before the MCT-encoding task (Figure 1A). This practice session allowed the participants to become familiarized with the task and helped to attenuate primacy effects. To further balance remaining primacy effects across participants, 10 trials from each retrieval condition (i.e., to-be intact or to-be rearranged) were randomly selected and presented in an intermixed way in the first 20 trials and the same items were tested at the beginning of the MCT-recognition task (see below). Note that these 20 trials were not removed from analyses. We aimed to minimize primacy effects because the current study focused on the encoding process; primacy effects are mainly due to, for example, novelty effects during encoding. We did not make specific restrictions to control recency effects, but because trials were presented in a random order, no systematic influence on recency effects can be expected.

Within each trial, a 1-sec presentation of a context stimulus was followed by a 3-sec overlaid presentation of an oval-shaped face stimulus to allow the encoding of context alone without the interference from centrally presented face stimuli. Participants then reported how well they could imagine the person being part of this scene within $1 \mathrm{sec}$, using a four-item scale (i.e., from "not vivid at all" to "perfectly vivid"). This trial-by-trial index was used as subjective contextualization ability. Total duration of the MCT-encoding task was 14 min $(7 \mathrm{sec} \times 120$ trials $)$.

MCT-recognition. Face recognition took place outside the scanner approximately $20 \mathrm{~min}$, with a range of 1822 min, after participants had finished the encoding task. The 120 old faces from encoding were intermixed with 120 new faces (i.e., "lures"). Crucially, to assess contextdependent memory, half of the old faces were presented against the same context stimuli as in the encoding task (intact retrieval condition), whereas the other half were reshuffled and presented in different face-context combinations (rearranged retrieval condition). New faces were randomly combined with the 120 old contexts. Thus, during recognition, each context was presented twice in total. As explained above, the first 20 trials from the encoding phase were intermixed with 20 trials containing new faces (i.e., not presented in the encoding task) and presented at the beginning of recognition for each participant. The number of trials for each retrieval condition out of those 40 trials was counterbalanced: 10 trials from the intact condition, 10 trials from the rearranged condition (thus in total 20 trials with old faces), as well as 
20 trials from the "lure" condition. During the entire task, the trial sequence was pseudorandomized individually, whereby neither old nor new faces, nor the trials from the same condition were presented on more than two trials consecutively.

For each trial, the face stimulus was presented overlaid onto the context. A 6-point confidence rating scale was presented at the bottom of the screen (Figure 1B), with which participants indicated if the face was old or new and to what extent they were confident about their judgment $(1=$ absolutely sure it was a new face; $2=$ somewhat sure it was a new face; $3=$ guessing it was a new face; 4 = guessing it was an old face; 5 = somewhat sure it was an old face; $6=$ absolutely sure it was an old face). Trials were self-paced, and a fixed 2-sec ITI was used. On average, the MCT-recognition task took $22 \mathrm{~min}$ $(5.5 \mathrm{sec} \times 240$ trials $)$.

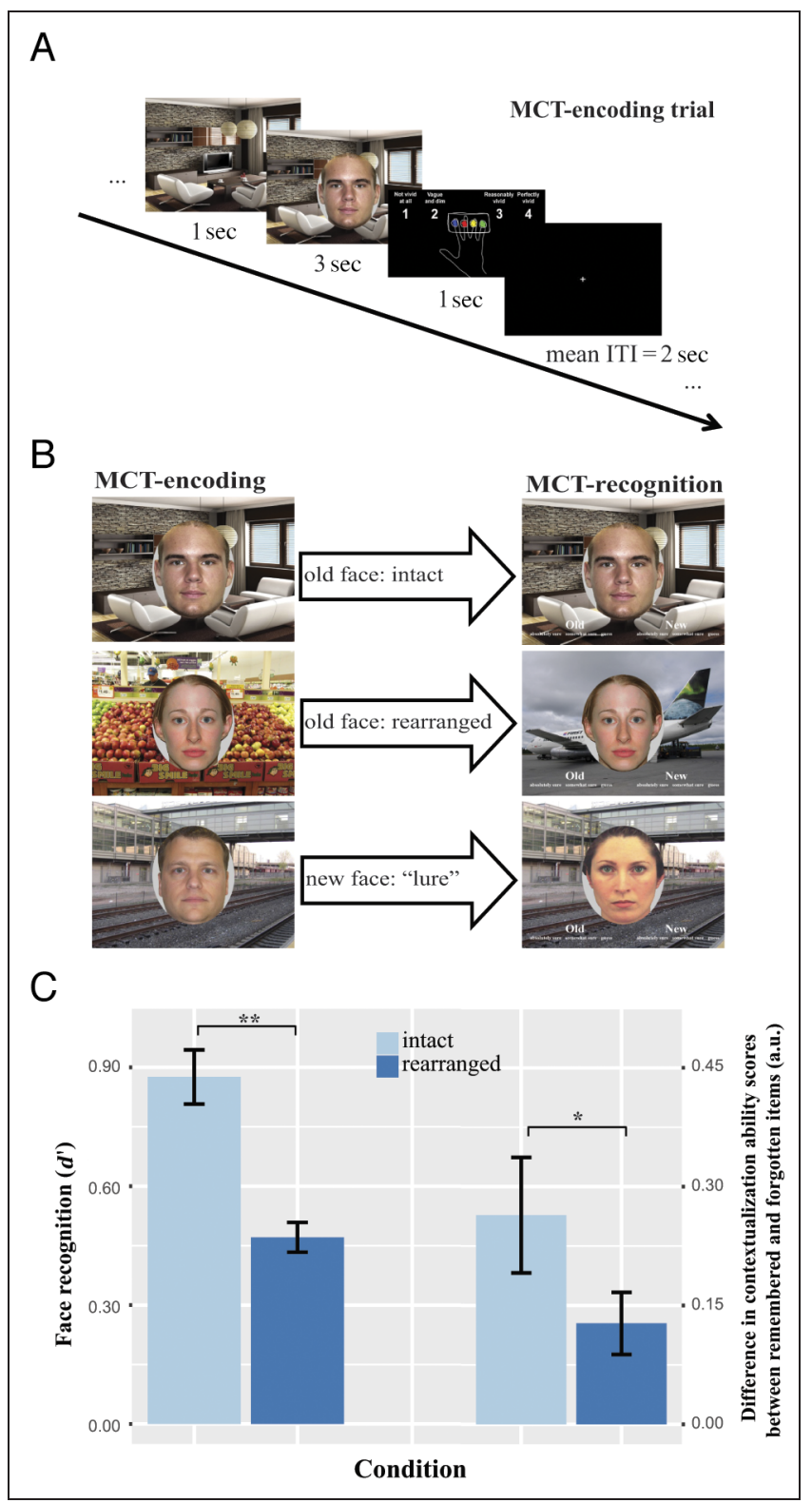

\section{Data Acquisition and Analysis}

\section{fMRI Data Acquisition}

All images were acquired using a 3-T Siemens Magnetom Skyra (Erlangen, Germany) MRI scanner with a 32-channel head coil at the Donders Institute for Brain, Cognition and Behaviour in Nijmegen, the Netherlands. High-resolution structural images $\left(1 \times 1 \times 1 \mathrm{~mm}^{3}\right)$ were acquired using a T1-weighted magnetization-prepared rapid gradient echo sequence (repetition time $[\mathrm{TR}]=2300 \mathrm{msec}$, echo time $[\mathrm{TE}]=3.03 \mathrm{msec}$, flip angle $=8^{\circ}$, field of view $[\mathrm{FOV}]=256 \times$ $256 \times 192 \mathrm{~mm}^{3}$ ). During both localizer and encoding tasks, T2*-weighted dual-echo EPI BOLD-fMRI images were acquired using an interleaved ascending slice acquisition sequence (slices $=40, \mathrm{TR}=2570 \mathrm{msec}$, TEs $=$ $15 / 35.7 \mathrm{msec}$, flip angle $=90^{\circ}$, voxel size $=2 \times 2 \times 2 \mathrm{~mm}^{3}$, slice gap $=0.34 \mathrm{~mm}$, FOV $=212 \times 212 \mathrm{~mm}^{2}$ ). Gradientecho field-map data were also acquired for EPI off-resonance distortion correction (slices $=64, \mathrm{TR}=1020 \mathrm{msec}$, TEs $=$ $10 / 12.46 \mathrm{msec}$, flip angle $=90^{\circ}, \mathrm{FOV}=224 \times 224 \mathrm{~mm}^{2}$, slice thickness $=2 \mathrm{~mm}$ ).

\section{Behavioral Data Analysis}

Participants were tested in a within-subject factorial design with subsequent memory (later remembered vs. later forgotten) and retrieval context (intact vs. rearranged) as main experimental factors.

To ensure memory performance was above chance level, we conducted binomial tests to investigate the statistical significance of the observed deviations (number of remembered vs. forgotten trials) from the null distribution (i.e., random performance). We defined abovechance level performance as a number of correct trials

Figure 1. Experimental paradigm of MCT and the validation of the experiment. (A) MCT-encoding trial: For each trial of the encoding task, the context stimulus was presented for $1 \mathrm{sec}$, followed by the combined presentation of the face and context stimuli for $3 \mathrm{sec}$. Participants were instructed to imagine the person interacting with the context. Participants then reported how well they could imagine this interactive scene on a 4 -point scale $(1=$ not vivid at all, $4=$ perfectly vivid $)$ presented for $1 \mathrm{sec}$. Trials were separated by a jittered ITI with an average of $2 \mathrm{sec}$. (B) MCT-encoding and recognition: During recognition, previously presented face stimuli from the encoding task (left) were presented against either their original encoding context stimuli ("intact") or against different ones ("rearranged"). New face stimuli (not seen during encoding) were presented in "lure" trials against context stimuli (seen during encoding and recognition; bottom right). The presentation of face and context stimuli in the recognition task was self-paced. Participants were instructed to indicate if the presented face was old or new and to what extent they were confident about their judgment based on a 6-point scale. (C) A significant difference in $d^{\prime}$ was found between intact and rearranged trials, which validated the experimental manipulation of context dependency of face memory (left); higher subjective contextualization ability scores were found for remembered versus forgotten items in intact versus rearranged conditions (right). Error bars represent standard errors of the mean. $* * p<.001$, two-tailed; $* p<.05$, one-tailed. 
that has a chance of $p<.05$ of arising from this null distribution. This criterion led to a threshold of 134 correct trials ( $p=.041$ ) out of 240 faces presented in the recognition task. Based on this procedure, data from five participants were excluded from all analyses.

To assess memory recognition, hit rates (i.e., proportion of correct responses to "old" faces) and false alarm rates (i.e., proportion of incorrect responses to "new" faces) were calculated and then converted to $d^{\prime}$ (the sensitivity index used in signal detection theory that takes into account response bias (Emmerich, 1967) as a function of retrieval context and of subjective contextualization ability. The difference of $d^{\prime}$ between intact and rearranged conditions (i.e., delta $d^{\prime}$ ) quantified context dependency of memory, with a larger delta $d^{\prime}$ indicating a stronger contextualization effect.

Subjective contextualization ability was derived from the trial-by-trial vividness scores that participants reported for their imagined scenarios involving the face-in-context stimuli. These contextualization ability scores were analyzed using a repeated-measures ANOVA with Subsequent memory performance (i.e., hits vs. misses) and Retrieval conditions (i.e., intact vs. rearranged) as within-subject factors. When the analysis returned a significant interaction effect, we used a (one-tailed) $t$ test to test our prediction that the difference between contextualization ability scores for hits and misses would be larger in intact than in rearranged trials. Furthermore, hit rates corrected for false alarm rates (i.e., hit rates minus false alarm rates) were modeled as a function of confidence level (i.e., low, middle and high) and retrieval conditions (i.e., intact vs. rearranged) to investigate the association between memory performance and confidence rating-based memory strength (Kirwan, Wixted, \& Squire, 2008; Slotnick \& Dodson, 2005). A repeated-measures ANOVA was used for these models.

\section{fMRI Data Analysis}

Data preprocessing and statistical analyses were carried out using Statistical Parametric Mapping Software (SPM8, Wellcome Trust Centre for Neuroimaging). Before preprocessing, dual-echo images were corrected for geometric distortions caused by magnetic field inhomogeneity (Hutton et al., 2002) separately for each echo using field map images. The corrected single-echo images were then recombined using the parallel-acquired inhomogeneitydesensitized method (Poser, Versluis, Hoogduin, \& Norris, 2006). Preprocessing and further analyses were carried out on the combined images. The first five recombined EPI volumes were discarded to allow for T1 equilibration. Preprocessing of the fMRI data included coregistration of functional and structural images using mutual information maximization, spatial normalization with the Montreal Neurological Institute (MNI) template using nonlinear warping, and spatial smoothing using an 8-mm FWHM Gaussian kernel.
Functional localizer. To localize the brain regions responsive to faces and contexts respectively, brain activation in response to faces in contrast to scrambled faces, and to contexts in contrast to scrambled contexts, were estimated using a general linear model with 24 additional motion parameters as nuisance regressors (six realignment parameters, six squared realignment parameters, six first derivatives of realignment parameters, and six squared first derivatives of realignment parameters). Voxellevel whole-brain family-wise error (FWE) corrections were used as multiple comparison correction. Because the FG (including FFA) and the amygdala have been implicated in facial feature processing (Mende-siedlecki, Said, \& Todorov, 2013; Todorov, 2012) whereas the PHG (including PPA) has been associated with context representations, we a priori hypothesized that these regions would be involved in face and context processing, respectively. In line with these hypotheses, we then created three spherical ROIs with an 8-mm radius centered at the peak voxels of aforementioned regions.

Memory contextualization. To investigate brain mechanisms underlying memory contextualization, all trials during encoding were sorted based on whether faces were later remembered or forgotten (i.e., SMEs; Paller \& Wagner, 2002; Kirchhoff, Wagner, Maril, \& Stern, 2000; Brewer, Zhao, Desmond, Glover, \& Gabrieli, 1998; Wagner, 1998; Gabrieli, Brewer, Desmond, \& Glover, 1997; Nyberg, Cabeza, \& Tulving, 1996). To ensure that neural correlates truly related to successful memory formation, we checked whether the subjective confidence levels as assessed during memory recognition related to successful subsequent memory. Hit rates differed from false alarm rates for high, $t(18)=5.45, p<.001$, middle, $t(19)=10.23, p<$ .001 , and low confidence levels, $t(19)=2.15, p=.045$. However, a closer examination revealed that, at the lowest confidence level, participants on average recognized old faces correctly in 21 out of a total 38 of trials (hit rate $=55 \%$ ), whereas they misjudged new faces as "old" (i.e., false alarm) in 19 trials out of a total of 40 (false alarm rate $=48 \%$ ). Based on the observed false alarm rate (19/40), one can estimate the number of correct guesses as $(38 \times 19 / 40=) 18$ trials out of a total of 38 low-confidence trials. Therefore, only $(21-18=)$ three additional correct recognitions on average can be attributed to a performance benefit due to memory. We therefore excluded all low-confidence correct trials from the SME analyses. The "remembered" trials for the SME analyses therefore only included trials where participants recognized faces with middle to high confidence level. Consequently, the average trial number for each regressor (i.e., hits/misses in intact/ rearranged conditions) in the fMRI statistical model ranged from 23 to 37 across participants.

For statistical analysis of the encoding task, event-related trial responses were modeled with 4-sec box-car functions in a first-level general linear model analysis and separate regressors were created for "remembered" and "forgotten" 
trials in intact versus rearranged conditions. In addition, subjective contextualization ability scores were added in the model as linear parametric modulators to remembered and forgotten trials, respectively, which we expected to predict the subsequent memory of faces. Mean time series of white matter and CSF as well as 24 motion parameters were included as nuisance regressors. The main effects of subsequent memory and their interactions with retrieval context (i.e., memory contextualization where SMEs differ between conditions) were tested at the group level. A priori ROIs, including the amygdala and face- and contextresponsive regions, were used for small volume correction (SVC). In addition to those functional ROI masks derived from the localizer task, we used the standardized bilateral hippocampus parcellation from the Automated Anatomical Labeling template (Tzourio-Mazoyer et al., 2002).

Finally, a psychophysiological interaction (PPI) analysis (Gitelman, Penny, Ashburner, \& Friston, 2003) was conducted to investigate functional connectivity associated with memory contextualization. The brain regions that were not only activated in response to the overall SME contrast (remembered vs. forgotten trials) but also associated with the subjective contextualization ability score were taken as the seed region for the analysis. We first extracted the BOLD time courses of the seed region and calculated the first eigenvariate. We then deconvolved this time course using the canonical hemodynamic response function to obtain the estimated time course of neural activity, which was used as physiological component. The interaction of the subsequent memory (remembered vs. forgotten) and retrieval context (intact vs. rearranged) was used to define the psychological component. We then created the PPI term by multiplying the psychological and physiological components. This interaction time course, which is used to test for stronger connectivity associated with subsequent memory in intact versus rearranged trials, was reconvolved with the canonical hemodynamic response function and then included in new first-level models alongside the first eigenvariate of the seed region time course. We then calculated the parameter estimate maps for the PPI regressor for each participant and used a one-sample $t$ test at second level to test this interaction at the group level. We further used the effective connectivity coefficients of this PPI analysis to behaviorally predict context dependency of memories that was indicated by the delta $d^{\prime}$ between intact and rearranged conditions across all participants via an ANCOVA. We further checked the distribution of delta $d^{\prime}$ based on the calculation of Mahalanobis distance for potential outliers and then used nonparametric permutation tests (Nichols \& Holmes, 2001) for verification of results if any data point deviated from the mean more than two standard deviations.

All statistical analyses of fMRI data have used voxel-level whole-brain FWE corrections or SVCs for a priori ROIs with $p<.05$.

\section{RESULTS \\ Memory Performance}

As expected, we found stronger context dependency of memory for faces, indicated by higher $d^{\prime}$ in intact versus rearranged trials, $F(1,20)=64.59, \eta_{p}^{2}=.76, p<.001$ (see Figure 1C; also see hits/misses per condition, per confidence level in Table 1). Confidence ratings during recognition, which can be seen as a measure of memory strength (Kirwan et al., 2008; Slotnick \& Dodson, 2005), were associated with memory performance, $F(2,26)=$ $156.11, \eta_{p}^{2}=.92, p<.001$, as indicated by a main effect of Confidence level on memory performance, with better memory performance (i.e., higher hit rate minus false alarm rate) at higher confidence levels: high level $>$ middle level, $t(14)=11.7, p<.001 ;$ middle level $>$ low level, $t(16)=$ $7.99, p<.001$. This effect was also modulated by context, $F(1,13)=7.84, \eta_{p}^{2}=.38, p<.005$, as indicated by an interaction effect of Confidence level and Retrieval condition, with all three levels of confidence ratings associated with better memory performance in intact versus rearranged

Table 1. Descriptives [Mean Number of Trials and Accuracy (SEM)] of Memory Performance

\begin{tabular}{|c|c|c|c|c|c|c|c|c|}
\hline \multirow{3}{*}{$\frac{\text { Stimulus }}{\text { Retrieval Context }}$} & \multicolumn{6}{|c|}{ Target } & \multirow{2}{*}{\multicolumn{2}{|c|}{$\begin{array}{l}\text { Lure } \\
\text { n.a. }\end{array}$}} \\
\hline & \multicolumn{3}{|c|}{ Intact } & \multicolumn{3}{|c|}{ Rearranged } & & \\
\hline & Hit & Miss & $H R-F A R$ & Hit & Miss & $H R-F A R$ & $C R$ & $F A$ \\
\hline \multicolumn{9}{|l|}{ Confidence level } \\
\hline Overall & 39.57 (1.41) & $20.86(1.43)$ & $0.16(0.03)$ & 30.05 (1.08) & $29.52(1.04)$ & $0.03(0.02)$ & 80.86 (2.18) & $39.14(2.18)$ \\
\hline High & 16.67 (2.14) & $5.71(1.36)$ & $0.54(0.03)$ & $8.19(1.28)$ & 7.57 (1.69) & $0.33(0.05)$ & $24.71(4.63)$ & $6.95(1.33)$ \\
\hline Middle & $13.14(1.43)$ & $8.24(0.79)$ & $0.10(0.03)$ & 11.57 (1.13) & $11.76(1.14)$ & $0(0.03)$ & $35.62(3.10)$ & $13.38(1.52)$ \\
\hline Low & $9.76(1.28)$ & $6.90(1.08)$ & $-0.17(0.04)$ & $10.29(1.08)$ & $10.19(1.28)$ & $-0.24(0.03)$ & $20.52(2.29)$ & $18.81(1.83)$ \\
\hline$d^{\prime}$ & \multicolumn{2}{|c|}{$0.88(0.07)$} & n.a. & \multicolumn{2}{|c|}{$0.47(0.04)$} & n.a. & \multicolumn{2}{|c|}{ n.a. } \\
\hline
\end{tabular}

Note that false alarm rates are assessed in lure trials and can therefore not be calculated separately for intact versus rearranged trials. CR $=$ correct rejection; $\mathrm{FA}=$ false alarm; HR-FAR $=$ hit rate minus false alarm rate; n.a. $=$ not applicable. 
Table 2. Descriptives [Mean Scores $(S E M)$ ] of Subjective Contextualization Ability

\begin{tabular}{|c|c|c|c|c|}
\hline \multirow{2}{*}{$\frac{\text { Retrieval Context }}{\text { Memory Performance }}$} & \multicolumn{2}{|c|}{ Intact } & \multicolumn{2}{|c|}{ Rearranged } \\
\hline & Hit & Miss & Hit & Miss \\
\hline Overall & $2.90(.075)$ & $2.70(.087)$ & $2.88(.080)$ & $2.75(.087)$ \\
\hline High confidence level & $3.09(.11)$ & $2.13(.33)$ & $2.79(.18)$ & $2.31(.25)$ \\
\hline Middle confidence level & $2.65(.17)$ & $2.54(.17)$ & $2.65(.17)$ & $2.47(.18)$ \\
\hline Low confidence level & $2.41(.20)$ & $2.42(.20)$ & $2.67(.18)$ & $2.67(.18)$ \\
\hline
\end{tabular}

Note that subjective contextualization ability score was not tested statistically as a function of confidence level.

trials: intact $_{\text {high }}>$ rearranged $_{\text {high }}, t(15)=6.14, p<.001$; intact $_{\text {mid }}>$ rearranged $_{\text {mid }}, t(17)=3.84, p<.005$; intact $_{\text {low }}>$ rearranged $_{\text {low }}, t(16)=2.29, p<.05$ (also see Table 1$)$.

Higher subjective contextualization ability scores during encoding were associated with trials that were later remembered versus forgotten, $F(1,20)=19.55, \eta_{p}^{2}=.49$, $p<.001$ (mHit $=2.84$, SEM $=0.44 ;$ mMiss $=2.64$, SEM $=$ $0.46)$. In agreement with our expectations, this difference in subjective contextualization ability score for remembered versus forgotten items was enhanced by context similarity, $t(20)=1.79, p=.045$, one-tailed (see Figure $1 \mathrm{C}$ and Table 2), suggesting a predictive effect of contextualization ability on the degree to which context aids retrieval.

These behavioral results demonstrate that the MCT resulted in context-dependent memories, allowing to then investigate the neural mechanisms of these effects.

\section{Definition of ROIs}

Brain activity during the localizer task was investigated using the [face $>$ scrambled face] and [context $>$ scrambled context] contrasts. Among other regions, the FFA, the amygdala, and the inferior frontal gyrus (IFG) were identified for the contrast [Face $>$ Scrambled Face]. A cluster of suprathreshold voxels lying at the junction of the FG and PHG (FG-PHG junction) for the contrast [Context $>$ Scrambled Context] was detected as well (see Table 3 for full results). Specifically, as one of the most active clusters in response to contexts resided in the FG and extended to the adjacent regions (i.e., PHG), the peak voxel in the FG-PHG junction was defined as the voxel with strongest activity within the PHG that was closest to the boundaries of two regions based on Automated Anatomical Labeling atlas. For later analyses on these ROIs, spherical functional ROI masks for FFA, amygdala, and FG-PHG junction were created on the basis of these results, in addition to the anatomical template of the hippocampus (see Memory Contextualization section).

\section{Memory Contextualization}

Brain regions associated with SMEs independent of context (i.e., all remembered $>$ all forgotten) were identified in the left IFG $(p<.05$, whole-brain FWE) and left amygdala $(p<.001$, SVC), but not in FFA and the FG-PHG junction (not even at a more liberal threshold of $p<$ .005 , uncorrected). A cluster of suprathreshold voxels in the left hippocampus showed marginally significant activation ( $p=.058$, SVC; see Figure 2A and Table 3).

We then checked which neural mechanisms at encoding might have mediated the subsequent contextualization memory benefit. We defined memory contextualization at the neural level as SMEs as a function of retrieval context (i.e., interaction between subsequent memory and retrieval context). No significant voxels emerged for this contrast. However, higher trial-by-trial subjective contextualization ability score, which was tested orthogonally to the memory effect (i.e., remembered vs. forgotten), was associated with enhanced activity in a cluster of suprathreshold voxels in the same left IFG region as revealed in the main SME $(p<.05$, FWE; see Table 3$)$. These findings reveal involvement of left IFG both in general memory performance as well as in subjective contextualization ability. These results raised the question whether IFG activity would still predict subsequent memory when controlling for subjective contextualization ability. We tested this effect in a separate model and observed the same IFG cluster (peak voxel: $-46,26,20)$ showed stronger activity in remembered versus forgotten trials.

Given our expectation that PFC plays a role in information integration as well as the association between the left IFG and both objective subsequent memory and subjective contextualization ability, we performed a left IFGbased PPI analysis to investigate if the left-IFG based functional connectivity could potentially explain context dependency of memories. Enhanced connectivity between the left IFG and left amygdala indeed predicted stronger subsequent memory for trials in the intact versus the rearranged condition ( $p<.05$, SVC; see Figure $2 \mathrm{~B})$. Connectivity with the right FG-PHG junction showed a trend in the same direction $(p=.054$, SVC; see Figure 2B). We then investigated whether the effective connectivity between these regions would also predict interindividual differences in context-dependent face memory performance. The two PPI-derived neural coupling estimates were added as covariates in separate 
Table 3. Peak Voxel Coordinates in MNI Space and $t$ Statistics

\begin{tabular}{|c|c|c|c|c|c|c|c|c|}
\hline \multirow{2}{*}{$\frac{\text { Contrast }}{\text { Region }}$} & \multicolumn{4}{|c|}{ Left Hemisphere } & \multicolumn{4}{|c|}{ Right Hemisphere } \\
\hline & $x$ & $y$ & $z$ & Peak $(t)$ & $x$ & $y$ & $z$ & Peak (t) \\
\hline \multicolumn{9}{|l|}{ Localizer } \\
\hline \multicolumn{9}{|l|}{ Face $>$ scrambled face } \\
\hline Amygdala & -26 & -2 & -16 & $4.02 *$ & 22 & -2 & -18 & $5.29 *$ \\
\hline Fusiform gyrus & -36 & -48 & -20 & 11.23 & 44 & -44 & -22 & 11.73 \\
\hline Inferior occipital cortex & -44 & -80 & -8 & 10.24 & 46 & -80 & -10 & 15.87 \\
\hline IFG (opercular) & & & & & 40 & 12 & 26 & 5.54 \\
\hline IFG (orbital) & & & & & 36 & 28 & -18 & 6.27 \\
\hline IFG (triangularis) & -40 & 20 & 24 & 5.52 & 44 & 24 & 28 & 6.31 \\
\hline Mid occipital cortex & -34 & -86 & -8 & 6.26 & 32 & -86 & 6 & 5.55 \\
\hline Mid temporal lobe & -50 & -64 & 2 & 5.75 & 46 & -52 & 0 & 6.19 \\
\hline Superior temporal lobe & & & & & 54 & -42 & 16 & 5.93 \\
\hline Supramarginal gyrus & -64 & -50 & 28 & 5.48 & & & & \\
\hline \multicolumn{9}{|c|}{ Context $>$ scrambled context } \\
\hline Calcarine sulcus & -18 & -62 & 12 & 8.49 & 24 & -58 & 20 & 7.98 \\
\hline Cerebellum & -30 & -40 & -24 & 6.23 & & & & \\
\hline Fusiform gyrus & -26 & -44 & -10 & 11.68 & 30 & -42 & -8 & 13.8 \\
\hline Inferior occipital cortex & -42 & -76 & -10 & 6.56 & 44 & -82 & -8 & 7.18 \\
\hline Lingual gyrus & -16 & -50 & 4 & 5.47 & 16 & -52 & 4 & 6.77 \\
\hline Mid occipital cortex & -34 & -88 & 12 & 12.04 & 36 & -82 & 18 & 11.27 \\
\hline FG-PHG junction & -30 & -42 & -8 & $11.01^{\mathrm{a}}$ & 26 & -38 & -10 & $7.3^{\mathrm{a}}$ \\
\hline
\end{tabular}

\section{Encoding}

SMEs (remembered $>$ forgotten)

$\begin{array}{lrrrr}\text { Left IFG } & -44 & 24 & 20 & 5.73 \\ \text { Left amygdala } & -20 & -4 & -14 & 4.2 * * \\ \text { Left hippocampus } & -26 & -12 & -10 & 3.75^{*}\end{array}$

Subjective contextualization ability (main effect)

$\begin{array}{lllll}\text { Left IFG (triangularis) } & -46 & 24 & 18 & 3.75\end{array}$

Memory contextualization (PPI, SME in intact $>$ SME in rearranged)

\begin{tabular}{lrrrl} 
(left IFG-) Left amygdala & -26 & 4 & -22 & $4.85 * *$ \\
(left IFG-) FG/PHG junction & 24 & -42 & -16 & $3.99 *$ \\
\hline
\end{tabular}

All statistical values reported here were significant at $p<.05$, whole-brain FWE-corrected, unless indicated otherwise.

${ }^{a}$ Identified based on voxel intensity and distance to the cluster peak (see Results: Definition of ROIs).

$* p<.05$, small volume corrected.

$* * p<.01$, small volume corrected. 
Figure 2. Brain activity associated with SMEs and contextualization. (A) SMEs were found in the left IFG (left) and left amygdala (middle); a small cluster of suprathreshold voxels in the left hippocampus (right) also showed marginally significant activity. (B) Stronger neural coupling between the left IFG (seed region) and the left amygdala (left) was associated with memory contextualization (greater SME in intact vs. rearranged conditions); neural coupling between the left IFG (seed region) and the right FG-PHG junction was marginally significant for the same contrast (right). The images are thresholded at $p<.001$ uncorrected, for visualization purposes. Peak voxels of clusters assigned to the left IFG, the amygdala, and the hippocampus (see Table 3) fell within these regions as defined by the Automatic Anatomical Labeling template. The left IFG cluster is located in the triangular part.

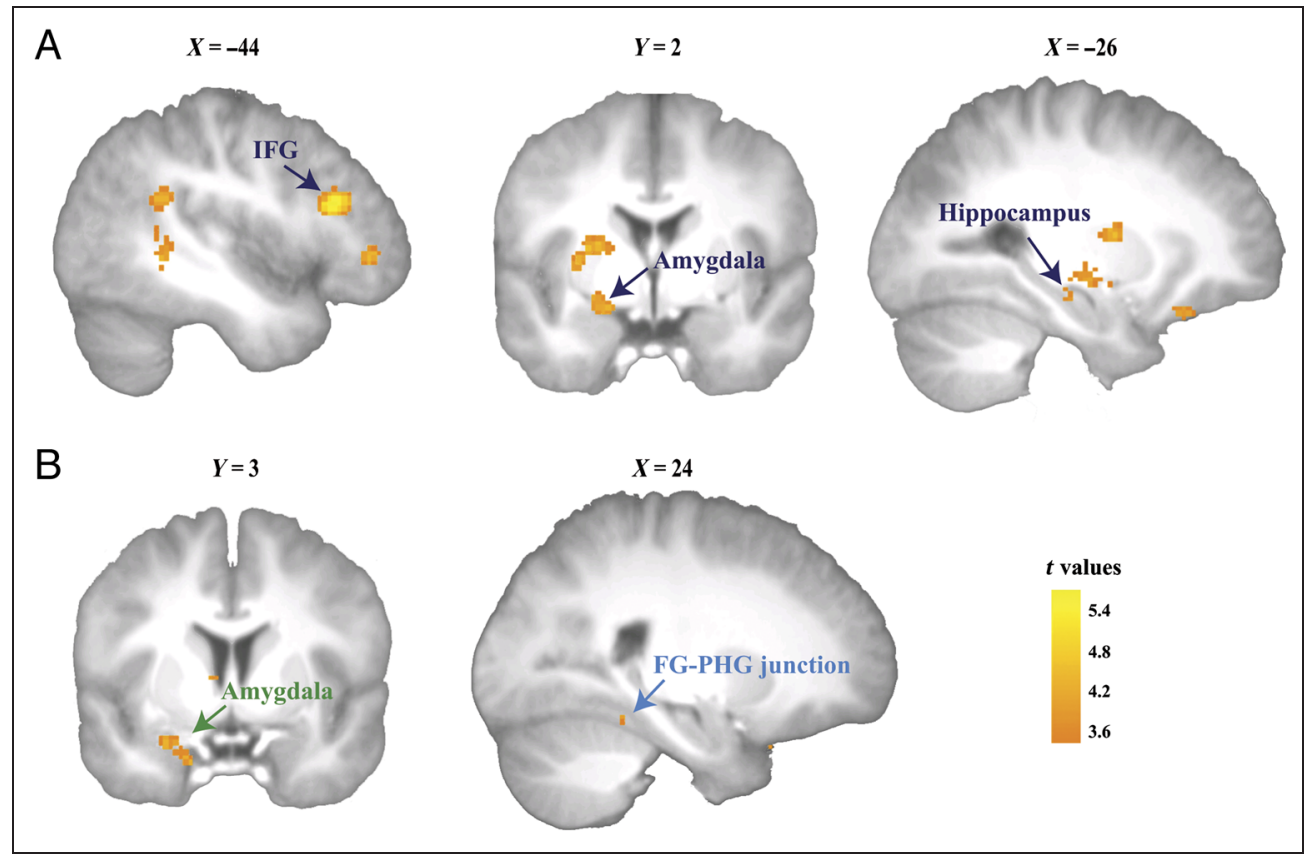

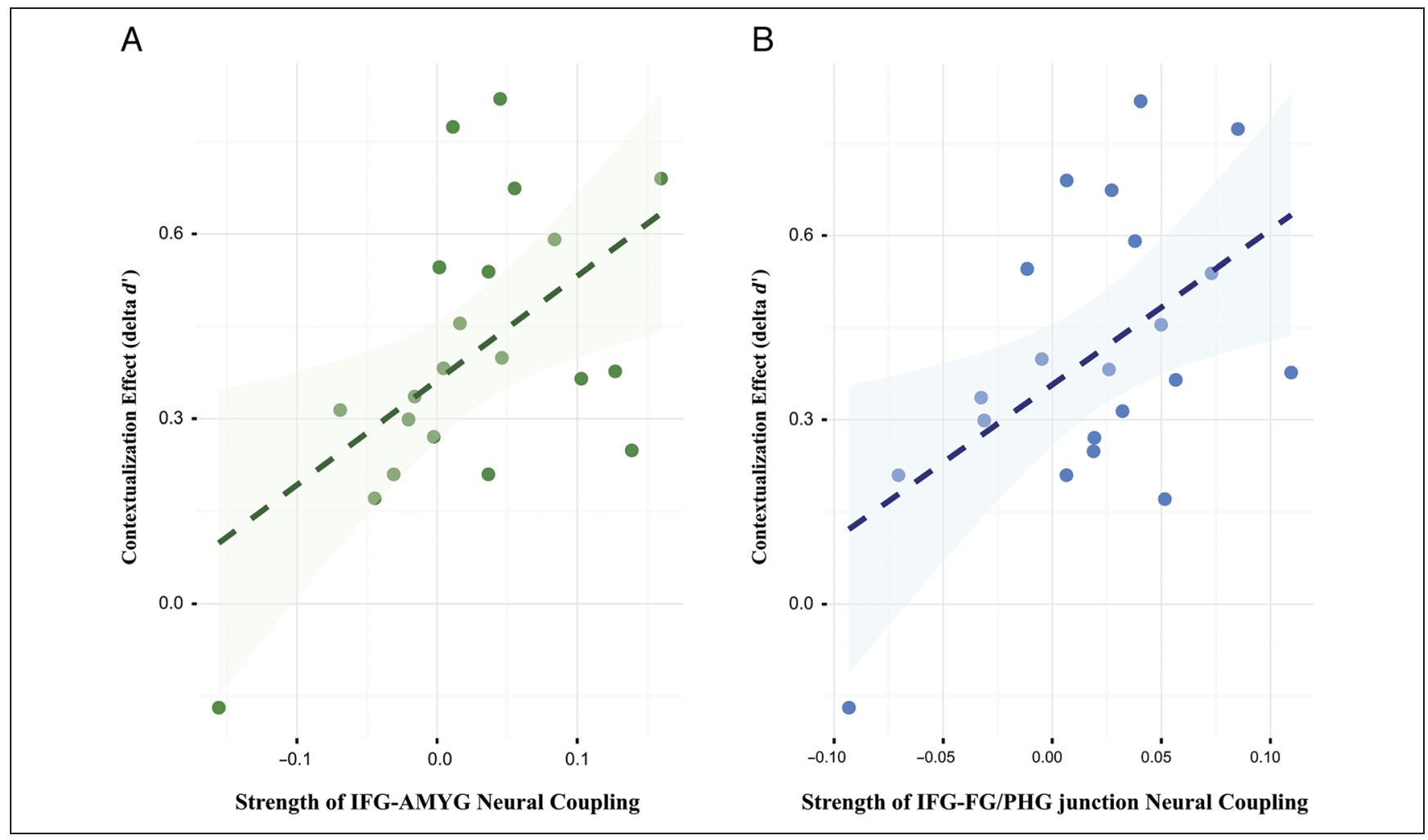

Figure 3. Neural coupling predictive of context-dependent memories. (A) The strength of neural coupling between the left IFG and amygdala predicted interindividual differences in context dependency of memory at the behavioral level, as indicated by differences in $d^{\prime}$ between intact and rearranged retrieval conditions (delta $d^{\prime}$ ). (B) The strength of neural coupling between the left IFG and FG-PHG junction also predicted interindividual differences in context dependency of memory. 
ANCOVAs with Retrieval context as within-subjects factor and Memory performance as dependent variable. We found significant interactions between Retrieval context and the left IFG-amygdala connectivity, $F(1,19)=7.85$, $\eta_{p}^{2}=.29, p<.05$ (see Figure 3A), and between Retrieval context and the left IFG-FG/PHG junction connectivity, $F(1,19)=7.57, \eta_{p}^{2}=.29, p<.05$ (see Figure 3B). Nonparametric correlation tests with 100,000 random permutations further confirmed these findings (IFG-amygdala: $r(19)=0.54, p=.012$; IFG-FG/PHG junction: $r(19)=$ $0.53, p=.013)$. Together, these additional tests confirm that stronger left IFG-based coupling with the amygdala and with the FG-PHG junction, all associated with memory contextualization processes, predict stronger contextdependent memory, both within and between participants.

\section{DISCUSSION}

The ability to store memories in conjunction with a representation of their encoding context may protect against subsequent dysfunctional memory generalization. Therefore, the current study aimed to reveal the functional neurobiology by which the brain contextualizes biologically meaningful items during encoding, resulting in subsequent context-dependent memories. Indeed, recognition performance was enhanced when the retrieval context was identical to the original encoding context, suggesting effective memory contextualization processes during encoding. Context-dependent memories were associated with stronger neural coupling during encoding between the left IFG and the amygdala, as well as between the left IFG and a region at the junction of FG and PHG. Importantly, the strength of these neural connections also predicted the extent of the context dependency of memories when tested across participants, providing additional evidence that contextualization processes during encoding are mediated by these regions.

In the current study, we used an incidental memory test to investigate how item recognition memory, in this case for faces, is facilitated by contextual information. This contrasts our study with previous work on relational and associative memory, which has provided important insight into neural mechanisms underlying item binding but has commonly used explicit memory tests involving recognition of pairs of items (Davachi, 2006). The advantage of testing implicit context effects on item memory during recognition, combined with incidental encoding of items-in-contexts, is that it most closely mimics real-life memory function: Context-dependent memory is considered a hallmark of human memory function (Tulving \& Thomson, 1973). Notably, contemporary animal research shows that, when confronted with a familiar context, hippocampal context codes are automatically reexpressed, thereby priming the relevant memories and reducing the interference from memories associated with other contexts (for a review, see Smith \& Bulkin, 2014): There seems to be no need to explicitly encode or assess the association of the item with its context. Thus, our paradigm builds on a long-standing tradition of both animal and human behavioral work showing the power of context to (implicitly) aid memory function.

The two studies by Hayes et al. (2007, 2010) did use a similar experimental setup as ours. However, their findings of the hippocampal and parahippocampal involvement could be explained by processing of more complex visual features due to unbalanced visual input in the contrast. To account for this, we adopted a task that was used in previous behavioral studies into the context dependency of memories. In this task, all items are encoded against context backgrounds, but $50 \%$ of contexts are rearranged during retrieval (van Ast et al., 2013, 2014). Unlike these studies, which used words as items, here we used faces because we specifically intended to investigate brain mechanisms by which emotional or biologically salient items are bound to contexts during encoding. An additional motivation was that faces are known to consistently activate a face-responsive region within the FG (Kanwisher et al., 1997) and the amygdala (Costafreda, Brammer, David, \& Fu, 2008; Sergerie, Chochol, \& Armony, 2008), a brain structure that is well known for its involvement in processing emotion and salience (Sergerie et al., 2008; Liberzon, Phan, Decker, \& Taylor, 2003). Because another area, located within the PHG (i.e., PPA), responds to the presentation of spatial scenes (Epstein et al., 1999), our design allowed us to distinguish item (i.e., face) and context (i.e., scene) representations at the neural level.

Behaviorally, our results revealed that memory recognition was strongly facilitated when the encoding and retrieval contexts were identical. This observation of context dependency of memories aligns well with previous studies using highly similar experimental paradigms (Meyer et al., 2017; Cox et al., 2014; van Ast et al., 2013, 2014; Staudigl \& Hanslmayr, 2013; Talamini et al., 2010). New was that we assessed the subjective ability to contextualize items on a trial-by-trial basis during encoding. We observed that better trial-by-trial contextualization ability yielded better subsequent item recognition performance. More importantly, the degree to which the original encoding context could help later retrieval was positively associated with this contextualization ability. This is in line with the notion that information presented in a more vivid and detailed manner helps to enhance future recall of that information (Buchsbaum, Lemire-Rodger, Fang, \& Abdi, 2012). Individual imagery ability, indicated by measures of the vividness of visual imagery, has likewise been suggested to positively affect memory (Baddeley \& Andrade, 2000; McKelvie, 1984; McKelvie \& Demers, 1979). The current findings are in line with these studies but also extend these by showing a predictive effect of contextualization ability on context dependency of memories. This finding suggests that stronger associative imagery during encoding particularly benefits later recognition memory performance in the presence of contextual cues. 
With respect to the neural correlates of memory contextualization, we could not identify regions in which the amplitude of regional BOLD responses was predictive of context-dependent memory. It thus appears that contextualization is not strongly predicted by the mere magnitude of neural responses in any specific brain region. Rather, functional coupling between the left IFG and the amygdala, as well as between left IFG and the FGPHG junction, was positively related to face recognition as a function of retrieval context. In addition, the neural coupling strength of these regions predicted individual differences in the extent of context-dependent memory. The encoding-related activity of the IFG, part of PFC, has been consistently reported as being associated with SMEs (Kim, 2011). More specifically, it seems to process both relational and item-specific information as part of the ventrolateral PFC (Blumenfeld, Parks, Yonelinas, \& Ranganath, 2011; Ranganath, 2010b; Murray \& Ranganath, 2007), to generate associations between items (Addis \& McAndrews, 2006; Uncapher \& Rugg, 2005), and to support the durability of episodic memories (Uncapher \& Rugg, 2005). Our findings broaden understanding of the mnemonic functions of this region by providing compelling evidence that the left IFG is a key region enabling effective memory contextualization.

The observation that IFG connectivity rather than regional activity was associated with contextualization points toward a role for the IFG in actively integrating information across distributed regions. These distant regions included the amygdala, a core face-responsive region (Mende-siedlecki et al., 2013; Costafreda et al., 2008) that processes information forwarded by more face-selective regions such as the FFA (Todorov, 2012). Here, we found left IFG-amygdala rather than IFG-FFA connectivity, suggesting that memory contextualization of biologically meaningful stimuli such as faces might require more salient feature processing that involves the amygdala. Left IFG connectivity with a region at the junction of PHG and FG was also predictive of memory contextualization. We first identified this region as responsive to the spatial contexts employed in our localizer task. Previous studies have found that an adjacent region within the PHG, the PPA, responds selectively to images of houses or buildings (Aminoff, Kveraga, \& Bar, 2013; Epstein \& Kanwisher, 1998). The difference between these findings and ours may be explained by the fact that we chose scene images with more complex features to increase distinctiveness. Together, these findings indicate that strongly contextualized memories result from an IFG-based coordination of mnemonic processes across distant regions representing distinct aspects of a memory, likely including perceptual features, spatiotemporal context, and motivational salience.

The hippocampus is also known to play a critical role in memory formation (Eichenbaum, 2000; Scoville \& Milner, 2000; Gabrieli, 1998; Squire \& Zola-Morgan, 1991) and has been highlighted as the "binding" center where features or elements of episodes and environments that are essential for recollection are bound together (Yonelinas, 2013). Here, we observed a marginally significant main effect of subsequent memory (SME) in the hippocampus, which is consistent with previous studies on its critical role in memory formation (Wagner, 1998). However, we did not find hippocampal involvement associated with memory contextualization. Perhaps the current experimental design was more likely to invoke an active and complex process of information integration that strongly depends on higher-order cognitive processes supported by prefrontal regions such as the IFG. The hippocampus, on the other hand, may become more important during memory consolidation, a process that persists well beyond the time of initial encoding (Knowlton \& Fanselow, 1998; McClelland, McNaughton, \& O'Reilly, 1995; Squire, 1992) that was not targeted by the current design. Alternatively, the absence of evidence for hippocampal involvement might result from decreased power due to interindividual variability in functional specialization along the longitudinal axis of the hippocampus (Strange, Witter, Lein, \& Moser, 2014; Poppenk, Evensmoen, Moscovitch, \& Nadel, 2013). Future studies should use tailored tasks focusing also on the post-encoding period to elucidate hippocampal involvement in memory contextualization with higher anatomical precision.

Inappropriate memory contextualization is considered a hallmark of traumatic memories (Brewin et al., 2010). For instance, low-level memory representations that are improperly contextualized are thought to contribute to memory flashbacks in PTSD (Acheson et al., 2012; Liberzon \& Sripada, 2008; Ehlers \& Clark, 2000). The brain structures we identified as being involved in memory contextualization are especially sensitive to stress-related neuromodulatory changes (Arnsten, 2009, 2015; Hermans, Henckens, Joëls, \& Fernández, 2014). Stress levels of noradrenergic activation lead to occupation of lower-affinity alpha-1 adrenoceptors in PFC, thus impairing functioning of this region (Birnbaum, Gobeske, Auerbach, Taylor, \& Arnsten, 1999). Acute stress has an opposite effect on functioning of the amygdala, where beta- 1 adrenoceptors become engaged at elevated levels of noradrenergic activity (Arnsten, 2000). Such dual effects are thought to be amplified by glucocorticoid activation (Roozendaal, McEwen, \& Chattarji, 2009). In agreement, a previous study by van Ast et al. (2013) using a similar task as the current study indeed found impaired memory contextualization after a pharmacological elevation of glucocorticoid levels. Notably, reduced memory contextualization, as assessed by the same task as used in this study, has been shown to predict more traumatic memory intrusions and related distress upon seeing a "trauma movie" (trauma analogue in the lab; Meyer et al., 2017). These studies, together with the present findings, suggest that extreme stress associated with traumatic events could lead to unusually strong amygdala-based representations, whereas a transient suppression of PFC functioning may give rise to a lack of 
mnemonic integration, resulting in decontextualization, fragmentation, and generalization of memories. This interpretation is in line with the revised dual representation theory of PTSD, which states that traumatic experiences can be stored as sensory-bound memories isolated from original encoding contexts, which would allow for the retrieval of traumatic memories triggered by perceptual cues reminiscent of the original trauma without retrieval of the appropriate context (Bisby, Horner, Hørlyck, \& Burgess, 2016; Brewin et al., 2010). Our data, however, indicate that this model may place too much emphasis on hippocampal instead of PFC dysfunction as central factor (Diamond, Campbell, Park, Halonen, \& Zoladz, 2007). It should be noted that, in the current study, we only used neutral faces as item stimuli to investigate memory contextualization. Given the aversive and negatively arousing properties of traumatic events, studies directly manipulating the emotional valence and arousal of items are required to further explore the clinical implications of our findings regarding the neural substrates of memory contextualization. In addition, some studies have shown that emotional valence of the encoding context can influence memory retrieval that seems to recruit similar neural circuits activated during encoding (Hofstetter, Achaibou, \& Vuilleumier, 2012; Sterpenich et al., 2006; Erk, Martin, \& Walter, 2005; Erk et al., 2003). It is therefore important to investigate how emotional valence of contexts could contribute to inappropriate memory contextualization as well.

To conclude, our results indicate that memory contextualization depends on the integration of information across neural circuits that are involved in item and context representations. Our findings in particular highlight a key role for the left IFG in coordinating this mnemonic process. Given the vulnerability of the prefrontal regions to the effects of acute stress, our findings thereby provide a novel framework for understanding the pathogenesis of traumatic memories that are often seen in stress-related disorders such as PTSD.

\section{Acknowledgments}

This research was supported by a grant from the Netherlands Organization for Scientific Research (NWO) awarded to K. R. (016 085 354). E. J. H. was supported by a grant from the European Research Council (ERC-2015-CoG 682591), and V. v. A. was supported by a VENI NWO grant (451-16-021). We are especially thankful to Milou Sep for her help in piloting the MCT.

Reprint requests should be sent to Wei Zhang, Centre for Cognitive Neuroimaging, Donders Institute for Brain Cognition and Behaviour, Radboud Universiteit, Kapittelweg 29, 6525 EN Nijmegen, The Netherlands, or via e-mail:w.zhang@donders.ru.nl.

\section{REFERENCES}

Acheson, D. T., Gresack, J. E., \& Risbrough, V. B. (2012). Hippocampal dysfunction effects on context memory: Possible etiology for posttraumatic stress disorder. Neuropharmacology, 62, 674-685.
Addis, D. R., \& McAndrews, M. P. (2006). Prefrontal and hippocampal contributions to the generation and binding of semantic associations during successful encoding. Neuroimage, 33, 1194-1206.

Aminoff, E. M., Kveraga, K., \& Bar, M. (2013). The role of the parahippocampal cortex in cognition. Trends in Cognitive Sciences, 17, 379-390.

Arnsten, A. F. T. (2000). Through the looking glass: Differential noradenergic modulation of prefrontal cortical function. Neural Plasticity, 7, 133-146.

Arnsten, A. F. T. (2009). Stress signalling pathways that impair prefrontal cortex structure and function. Nature Reviews Neuroscience, 10, 410-422.

Arnsten, A. F. T. (2015). Stress weakens prefrontal networks: Molecular insults to higher cognition. Nature Neuroscience, 18, 1376-1385.

Baddeley, A. D., \& Andrade, J. (2000). Working memory and the vividness of imagery. Journal of Experimental Psychology: General, 129, 126-145.

Birnbaum, S., Gobeske, K. T., Auerbach, J., Taylor, J. R., \& Arnsten, A. F. T. (1999). A role for norepinephrine in stressinduced cognitive deficits: Alpha-1-adrenoceptor mediation in the prefrontal cortex. Biological Psychiatry, 46, 1266-1274.

Bisby, J. A., Horner, A. J., Hørlyck, L. D., \& Burgess, N. (2016). Opposing effects of negative emotion on amygdalar and hippocampal memory for items and associations. Social Cognitive and Affective Neuroscience, 11, 981-990.

Blumenfeld, R. S., Parks, C. M., Yonelinas, A. P., \& Ranganath, C. (2011). Putting the pieces together: The role of dorsolateral prefrontal cortex in relational memory encoding. Journal of Cognitive Neuroscience, 23, 257-265.

Brewer, J. B., Zhao, Z., Desmond, J. E., Glover, G. H., \& Gabrieli, J. D. (1998). Making memories: Brain activity that predicts how well visual experience will be remembered. Science, 281, 1185-1187.

Brewin, C. R., Gregory, J. D., Lipton, M., \& Burgess, N. (2010). Intrusive images in psychological disorders: Characteristics, neural mechanisms, and treatment implications.

Psychological Review, 117, 210-232.

Buchsbaum, B. R., Lemire-Rodger, S., Fang, C., \& Abdi, H. (2012). The neural basis of vivid memory is patterned on perception. Journal of Cognitive Neuroscience, 24, 1867-1883.

Chun, M. M., \& Phelps, E. (1999). Memory deficits for implicit contextual information in amnesic subjects with hippocampal damage. Nature Neuroscience, 2, 844-847.

Costafreda, S. G., Brammer, M. J., David, A. S., \& Fu, C. H. Y. (2008). Predictors of amygdala activation during the processing of emotional stimuli: A meta-analysis of 385 PET and fMRI studies. Brain Research Reviews, 58, 57-70.

Cox, R., Tijdens, R. R., Meeter, M. M., Sweegers, C. C. G., \& Talamini, L. M. (2014). Time, not sleep, unbinds contexts from item memory. PLOS ONE, 9, 1-6.

Davachi, L. (2006). Item, context and relational episodic encoding in humans. Current Opinion in Neurobiology, 16, 693-700.

Davachi, L., \& DuBrow, S. (2015). How the hippocampus preserves order: The role of prediction and context. Trends in Cognitive Sciences, 19, 92-99.

De Beni, R., \& Pazzaglia, F. (1995). Memory for different kinds of mental images: Role of contextual and autobiographic variables. Neuropsychologia, 33, 1359-1371.

Diamond, D. M., Campbell, A. M., Park, C. R., Halonen, J., \& Zoladz, P. R. (2007). The temporal dynamics model of emotional memory processing: A synthesis on the neurobiological basis of stress-induced amnesia, flashbulb and traumatic memories, and the Yerkes-Dodson law. Neural Plasticity, 2007, 60803.

Diana, R. A., Yonelinas, A. P., \& Ranganath, C. (2007). Imaging recollection and familiarity in the medial temporal lobe: A 
three-component model. Trends in Cognitive Sciences, 11, 379-386.

Ehlers, A., \& Clark, D. M. (2000). A cognitive model of posttraumatic stress disorder. Behaviour Research and Therapy, 38, 319-345.

Eichenbaum, H. (2000). A cortical-hippocampal system for declarative memory. Nature Reviews Neuroscience, 1, 41-50.

Eichenbaum, H. (2004). Hippocampus: Cognitive processes and neural representations that underlie declarative memory. Neuron, 44, 109-120.

Eichenbaum, H., Sauvage, M., Fortin, N., Komorowski, R., \& Lipton, P. (2012). Towards a functional organization of episodic memory in the medial temporal lobe. Neuroscience and Biobehavioral Reviews, 36, 1597-1608.

Emmerich, D. S. (1967). Signal detection theory and psychophysics. David M. Green, John A. Swets. The Quarterly Review of Biology, 42, 578.

Epstein, R., Harris, A., Stanley, D., \& Kanwisher, N. (1999). The parahippocampal place area: Recognition, navigation, or encoding? Neuron, 23, 115-125.

Epstein, R., \& Kanwisher, N. (1998). A cortical representation of the local visual environment. Nature, 392, 598-601.

Erk, S., Kiefer, M., Grothe, J., Wunderlich, A. P., Spitzer, M., \& Walter, H. (2003). Emotional context modulates subsequent memory effect. Neuroimage, 18, 439-447.

Erk, S., Martin, S., \& Walter, H. (2005). Emotional context during encoding of neutral items modulates brain activation not only during encoding but also during recognition. Neuroimage, 26, 829-838.

Gabrieli, J. D. E. (1998). Cognitive neuroscience of human memory. Annual Review of Psychology, 49, 87-115.

Gabrieli, J. D. E., Brewer, J. B., Desmond, J. E., \& Glover, G. H. (1997). Separate neural bases of two fundamental memory processes in the human medial temporal lobe. Science, 276, 264-266.

Giovanello, K. S., Schnyer, D. M., \& Verfaellie, M. (2004). A critical role of the anterior hippocampus in relational memory: Evidence from an fMRI study comparing associative and item recognition. Hippocampus, 14, 5-8.

Gitelman, D. R., Penny, W. D., Ashburner, J., \& Friston, K. J. (2003). Modeling regional and psychophysiologic interactions in fMRI: The importance of hemodynamic deconvolution. Neuroimage, 19, 200-207.

Godden, D. R., \& Baddeley, A. D. (1975). Context dependent memory in two natural environments: On land and underwater. British Journal of Psychology, 66, 325-331.

Graf, P., \& Schacter, D. L. (1985). Implicit and explicit memory for new associations in normal and amnesic subjects. Journal of Experimental Psychology: Learning, Memory, and Cognition, 11, 501-518.

Hayes, S. M., Baena, E., Truong, T.-K., \& Cabeza, R. (2010). Neural mechanisms of context effects on face recognition: Automatic binding and context shift decrements. Journal of Cognitive Neuroscience, 22, 2541-2554.

Hayes, S. M., Nadel, L., \& Ryan, L. (2007). The effect of scene context on episodic object recognition: Parahippocampal cortex mediates memory encoding and retrieval success. Hippocampus, 17, 873-889.

Hermans, E. J., Henckens, M. J. A. G., Joëls, M., \& Fernández, G. (2014). Dynamic adaptation of large-scale brain networks in response to acute stressors. Trends in Neurosciences, 37, 304-314.

Hofstetter, C., Achaibou, A., \& Vuilleumier, P. (2012). Reactivation of visual cortex during memory retrieval: Content specificity and emotional modulation. Neuroimage, 60, 1734-1745.

Hutton, C., Bork, A., Josephs, O., Deichmann, R., Ashburner, J., \& Turner, R. (2002). Image distortion correction in fMRI: A quantitative evaluation. Neuroimage, 16, 217-240.
Jackson, O., \& Schacter, D. L. (2004). Encoding activity in anterior medial temporal lobe supports subsequent associative recognition. Neuroimage, 21, 456-462.

Jenkins, L. J., \& Ranganath, C. (2010). Prefrontal and medial temporal lobe activity at encoding predicts temporal context memory. Journal of Neuroscience, 30, 15558-15565.

Kanwisher, N., McDermott, J., \& Chun, M. M. (1997). The fusiform face area: A module in human extrastriate cortex specialized for face perception. Journal of Neuroscience, 17, 4302-4311.

Kanwisher, N., \& Yovel, G. (2006). The fusiform face area: A cortical region specialized for the perception of faces. Philosophical Transactions of the Royal Society of London, Series B, Biological Sciences, 361, 2109-2128.

Kapur, S., Craik, F. I., Tulving, E., Wilson, A. A., Houle, S., \& Brown, G. M. (1994). Neuroanatomical correlates of encoding in episodic memory: Levels of processing effect. Proceedings of the National Academy of Sciences, U.S.A., 91, 2008-2011.

Kim, H. (2011). Neural activity that predicts subsequent memory and forgetting: A meta-analysis of 74 fMRI studies. Neuroimage, 54, 2446-2461.

Kirchhoff, B. A., Wagner, A. D., Maril, A., \& Stern, C. E. (2000). Prefrontal-temporal circuitry for episodic encoding and subsequent memory. Journal of Neuroscience, 20, 6173-6180.

Kirwan, C. B., Wixted, J. T., \& Squire, L. R. (2008). Activity in the medial temporal lobe predicts memory strength, whereas activity in the prefrontal cortex predicts recollection. Journal of Neuroscience, 28, 10541-10548.

Knowlton, B. J., \& Fanselow, M. S. (1998). The hippocampus, consolidation and on-line memory. Current Opinion in Neurobiology, 8, 293-296.

Langner, O., Dotsch, R., Bijlstra, G., Wigboldus, D. H. J., Hawk, S. T., \& van Knippenberg, A. (2010). Presentation and validation of the Radboud Faces Database. Cognition $\varepsilon$ Emotion, 24, 1377-1388.

Liberzon, I., Phan, K. L., Decker, L. R., \& Taylor, S. F. (2003). Extended amygdala and emotional salience: A PET activation study of positive and negative affect. Neuropsychopharmacology, 28, 726-733.

Liberzon, I., \& Sripada, C. S. (2008). The functional neuroanatomy of PTSD: A critical review. Progress in Brain Research, 167, 151-169.

Litman, L., \& Davachi, L. (2008). Distributed learning enhances relational memory consolidation. Learning \& Memory, 15, $711-716$

Long, N. M., \& Kahana, M. J. (2015). Successful memory formation is driven by contextual encoding in the core memory network. Neuroimage, 119, 332-337.

Lundqvist, D., Flykt, A., \& Ohman, A. (1998). The Karolinska directed emotional faces (KDEF). CD ROM from Department of Clinical Neuroscience, Psychology Section, Karolinska Institutet, 91-630. https://doi.org/10.1017/ S0048577299971664.

McClelland, J. L., McNaughton, B. L., \& O'Reilly, R. C. (1995). Why there are complementary learning systems in the hippocampus and neocortex: Insights from the successes and failures of connectionist models of learning and memory. Psychological Review, 102, 419-457.

McKelvie, S. J. (1984). Reported visual imagery for faces and facial recognition memory. Perceptual and Motor Skills, 59, 825-826.

McKelvie, S. J., \& Demers, E. G. (1979). Individual differences in reported visual imagery and memory performance. British Journal of Psychology, 70, 51-57.

Mende-siedlecki, P., Said, C. P., \& Todorov, A. (2013). The social evaluation of faces: A meta-analysis of functional neuroimaging studies. Social Cognitive and Affective Neuroscience, 8, 285-299.

Meyer, T., Krans, J., van Ast, V., \& Smeets, T. (2017). Visuospatial context learning and configuration learning is associated with 
analogue traumatic intrusions. Journal of Behavior Therapy and Experimental Psychiatry, 54, 120-127.

Meyer, T., Smeets, T., Giesbrecht, T., Quaedflieg, C. W. E. M., Girardelli, M. M., Mackay, G. R. N., et al. (2012). Individual differences in spatial configuration learning predict the occurrence of intrusive memories. Cognitive, Affective, $\varepsilon$ Behavioral Neuroscience, 13, 186-196.

Mumby, D. G., Gaskin, S., Glenn, M. J., Schramek, T. E., \& Lehmann, H. (2002). Hippocampal damage and exploratory preferences in rats: Memory for objects, places, and contexts. Learning \& Memory, 9, 49-57.

Murray, L. J., \& Ranganath, C. (2007). The dorsolateral prefrontal cortex contributes to successful relational memory encoding. Journal of Neuroscience, 27, 5515-5522.

Nichols, T. E., \& Holmes, A. P. (2001). Nonparametric permutation tests for $\{\mathrm{PET}\}$ functional neuroimaging experiments: A primer with examples. Human Brain Mapping, 15, 1-25.

Nyberg, L., Cabeza, R., \& Tulving, E. (1996). PET studies of encoding and retrieval: The HERA model. Psychonomic Bulletin \& Review, 3, 135-148.

Paller, K. A., \& Wagner, A. D. (2002). Observing the transformation of experience into memory. Trends in Cognitive Sciences, 6, 93-102.

Phillips, R. G., \& LeDoux, J. E. (1992). Differential contribution of amygdala and hippocampus to cued and contextual fear conditioning. Behavioral Neuroscience, 106, 274-285.

Poppenk, J., Evensmoen, H. R., Moscovitch, M., \& Nadel, L. (2013). Long-axis specialization of the human hippocampus. Trends in Cognitive Sciences, 17, 230-240.

Poser, B. A., Versluis, M. J., Hoogduin, J. M., \& Norris, D. G. (2006). BOLD contrast sensitivity enhancement and artifact reduction with multiecho EPI: Parallel-acquired inhomogeneity-desensitized fMRI. Magnetic Resonance in Medicine, 55, 1227-1235.

Prince, S. E. (2005). Neural correlates of relational memory: Successful encoding and retrieval of semantic and perceptual associations. Journal of Neuroscience, 25, 1203-1210.

Quaedflieg, C. W. E. M., van de Ven, V., Meyer, T., Siep, N., Merckelbach, H., \& Smeets, T. (2015). Temporal dynamics of stress-induced alternations of intrinsic amygdala connectivity and neuroendocrine levels. PLoS One, 10, e0124141.

Ranganath, C. (2010a). A unified framework for the functional organization of the medial temporal lobes and the phenomenology of episodic memory. Hippocampus, 20, 1263-1290.

Ranganath, C. (2010b). Binding items and contexts: The cognitive neuroscience of episodic memory. Current Directions in Psychological Science, 19, 131-137.

Richardson, J. T. E. (1980). Mental imagery and buman memory. London: Macmillan.

Rimmele, U., Davachi, L., \& Phelps, E. A. (2012). Memory for time and place contributes to enhanced confidence in memories for emotional events. Emotion, 12, 834-846.

Roozendaal, B., McEwen, B. S., \& Chattarji, S. (2009). Stress, memory and the amygdala. Nature Reviews Neuroscience, 10, 423-433.

Scoville, W. B., \& Milner, B. (2000). Loss of recent memory after bilateral hippocampal lesions. 1957. Journal of Neuropsychiatry and Clinical Neurosciences, 12, 103-113.

Sergerie, K., Chochol, C., \& Armony, J. L. (2008). The role of the amygdala in emotional processing: A quantitative meta-analysis of functional neuroimaging studies. Neuroscience and Biobehavioral Reviews, 32, 811-830.

Slotnick, S. D., \& Dodson, C. S. (2005). Support for a continuous (single-process) model of recognition memory and source memory. Memory \& Cognition, 33, 151-170.

Smith, D. M., \& Bulkin, D. A. (2014). The form and function of hippocampal context representations. Neuroscience and Biobehavioral Reviews, 40, 52-61.
Smith, S. M., \& Vela, E. (2001). Environmental context-dependent memory: A review and meta-analysis. Psychonomic Bulletin E Review, 8, 203-220.

Squire, L. (1992). Memory and the hippocampus: A synthesis from findings with rats, monkeys, and humans. Psychological Review, 99, 195-231.

Squire, L., \& Zola-Morgan, S. (1991). The medial temporal lobe memory system. Science, 253, 1380-1386.

Staudigl, T., \& Hanslmayr, S. (2013). Theta oscillations at encoding mediate the context-dependent nature of human episodic memory. Current Biology, 23, 1101-1106.

Sterpenich, V., D’Argembeau, A., Desseilles, M., Balteau, E., Albouy, G., Vandewalle, G., et al. (2006). The locus ceruleus is involved in the successful retrieval of emotional memories in humans. Journal of Neuroscience, 26, 7416-7423.

Strange, B., Witter, M. P., Lein, E. S., \& Moser, E. I. (2014). Functional organization of the hippocampal longitudinal axis. Nature Reviews Neuroscience, 15, 655-669.

Summerfield, C., Greene, M., Wager, T., Egner, T., Hirsch, J., \& Mangels, J. (2006). Neocortical connectivity during episodic memory formation. PLoS Biology, 4, 855-864.

Talamini, L. M., de Haan, L., Nieman, D. H., Linszen, D. H., \& Meeter, M. (2010). Reduced context effects on retrieval in first-episode schizophrenia. PloS One, 5, e10356.

Talamini, L. M., \& Gorree, E. (2012). Aging memories: Differential decay of episodic memory components. Learning \& Memory, 19, 239-246.

Todorov, A. (2012). The role of the amygdala in face perception and evaluation. Motivation and Emotion, 36, 16-26.

Tottenham, N., Tanaka, J. W., Leon, A. C., McCarry, T., Nurse, M., Hare, T. A., et al. (2009). The NimStim set of facial expressions: Judgments from untrained research participants. Psychiatry Research, 168, 242-249.

Tsivilis, D., Otten, L. J., \& Rugg, M. D. (2001). Context effects on the neural correlates of recognition memory: An electrophysiological study. Neuron, 31, 497-505.

Tulving, E. (1972). Episodic and semantic memory. In E. Tulving \& W. Donaldson (Eds.), Organization of memory (pp. 381-403). New York: Academic Press.

Tulving, E., \& Thomson, D. M. (1973). Encoding specificity and retrieval processes in episodic memory. Psychological Review, 80, 352-373.

Tzourio-Mazoyer, N., Landeau, B., Papathanassiou, D., Crivello, F., Etard, O., Delcroix, N., et al. (2002). Automated anatomical labeling of activations in SPM using a macroscopic anatomical parcellation of the MNI MRI single-subject brain. Neuroimage, 15, 273-289.

Uncapher, M. R., \& Rugg, M. D. (2005). Encoding and the durability of episodic memory: A functional magnetic resonance imaging study. Journal of Neuroscience, 25, 7260-7267.

van Ast, V., Cornelisse, S., Meeter, M., Joëls, M., \& Kindt, M. (2013). Time-dependent effects of cortisol on the contextualization of emotional memories. Biological Psychiatry, 74, 809-816.

van Ast, V., Cornelisse, S., Meeter, M., \& Kindt, M. (2014). Cortisol mediates the effects of stress on the contextual dependency of memories. Psychoneuroendocrinology, 41, 97-110.

Wagner, A. (1998). Prefrontal cortex and recognition memory. Functional-MRI evidence for context-dependent retrieval processes. Brain, 121, 1985-2002.

Yonelinas, A. P. (2013). The hippocampus supports highresolution binding in the service of perception, working memory and long-term memory. Behavioural Brain Research, 254, 34-44.

Zeidman, P., Mullally, S. L., \& Maguire, E. A. (2015). Constructing, perceiving, and maintaining scenes: Hippocampal activity and connectivity. Cerebral Cortex, 25, 3836-3855. 